\title{
Production of exotic isotopes in complete fusion reactions with radioactive beams
}

\author{
V.V. Sargsyan ${ }^{1,2}$, A.S. Zubov ${ }^{1}$, G.G. Adamian ${ }^{1}$, N.V. Antonenko ${ }^{1}$, and S. Heinz ${ }^{3}$ \\ ${ }^{1}$ Joint Institute for Nuclear Research, 141980 Dubna, Russia \\ ${ }^{2}$ International Center for Advanced Studies, \\ Yerevan State University, 0025 Yerevan, Armenia \\ ${ }^{3}$ GSI Helmholtzzentrum für Schwerionenforschung GmbH, 64291 Darmstadt, Germany
}

(Dated: November 7, 2018)

\begin{abstract}
The isotopic dependence of the complete fusion (capture) cross section is analyzed in the reactions $130,132,134,136,138,140,142,144,146,148,150 \mathrm{Xe}+{ }^{48} \mathrm{Ca}$ with stable and radioactive beams. It is shown for the first time that the very neutron-rich nuclei ${ }^{186-191} \mathrm{~W}$ can be reached with relatively large cross sections by complete fusion reactions with radioactive ion beams at incident energies near the Coulomb barrier. A comparison between the complete fusion and fragmentation reactions for the production of neutron-rich $\mathrm{W}$ and neutron-deficient $\mathrm{Rn}$ isotopes is performed.
\end{abstract}

PACS numbers: 25.70.Hi, 24.10.-i, 24.60.-k

Key words: Complete fusion reactions; Neutron-rich and neutron-deficient nuclei; Radioactive beams; Subbarrier capture 


\section{INTRODUCTION}

The new generation of radioactive ion beam facilities will provide high intensity $\left(>10^{9}\right.$ ions/s) exotic beams (for example, ${ }^{88-94} \mathrm{Kr},{ }^{126-132} \mathrm{Sn},{ }^{138-144} \mathrm{Xe}$ or ${ }^{119-132} \mathrm{Cs}$ ). One of the most interesting areas of research with radioactive beams will be the study of the complete fusion process [1] where the fusion experiments with exotic beams can be performed to synthesize and study new isotopes of existing elements. The central issue is whether the capture and fusion cross sections will be enhanced due to the large deformation of the neutron-rich or neutron-deficient projectile-nucleus. However, one should bear in mind the smaller intensity of these beams in comparison with the intensity of stable beams. Our aim is to find the global trend in the production cross section of exotic nuclei as a function of the charge (mass) number of the projectile in complete fusion reactions. Based on this trend one can find a consensus between the cross sections resulting from a certain beam and the intensity of this beam.

The goal of the present paper is to compare the fusion of stable ${ }^{130,132,134,136} \mathrm{Xe}$ and radioactive ${ }^{138,140,142,144,146,148,150} \mathrm{Xe}$ beams with the same target, ${ }^{48} \mathrm{Ca}$, in order to study the effects of the neutron excess and neutron transfer on the fusion process. The target ${ }^{48} \mathrm{Ca}$ is ideal for this purpose since this nucleus has the largest possible neutron excess and the systems ${ }^{138,140,142,144,146,148,150} \mathrm{Xe}+{ }^{48} \mathrm{Ca}$ have positive neutron transfer $Q$-values while all the corresponding reactions ${ }^{130,132,134,136} \mathrm{Xe}+{ }^{48} \mathrm{Ca}$ display negative $Q$-values. In the present paper we demonstrate for the first time the possibilities for producing neutron-rich isotopes of ${ }^{186-191} \mathrm{~W}$ in the complete fusion reactions ${ }^{146,148} \mathrm{Xe}+{ }^{48} \mathrm{Ca}$ with rather large cross sections.

The nucleus ${ }^{190} \mathrm{~W}$ was the heaviest isotope which has been synthesized in $(n, n 2 p)$ and $(p, 3 p)$ reactions [2]. In these experiments the chemical extraction of ${ }^{190} \mathrm{~W}$ was possible after long irradiation. Another method to produce the neutron-rich nuclei is fragmentation reactions [3, 4]. Cross-sections smaller than $0.4 \mu \mathrm{b}$ were measured for the isotopes ${ }^{190-192} \mathrm{~W}$ in cold fragmentation of $950 \mathrm{MeV} /$ nucleon ${ }^{197} \mathrm{Au}$ beams on Be targets [3]. However, the production cross section decreases strongly with increasing neutron number. The most neutron-rich $\mathrm{W}$ isotopes, up to ${ }^{197} \mathrm{~W}$, were observed in projectile fragmentation of ${ }^{238} \mathrm{U}$ at $1 \mathrm{GeV} /$ nucleon on Be targets at the Fragment Separator (FRS) at GSI [5]. Here, crosssections smaller than $5 \mathrm{nb}$ were measured for $\mathrm{W}$ isotopes with mass numbers $\mathrm{A} \geq 190$ where the cross-section decreases by approximately one order of magnitude for every two 
neutrons more in the residual nucleus. In the present paper we also compare the complete fusion reactions ${ }^{146} \mathrm{Xe}+{ }^{48} \mathrm{Ca}$ with fragmentation reactions leading both to the production of neutron-rich W isotopes. Additionally, we will compare the complete fusion reactions ${ }^{123} \mathrm{Cs}+{ }^{69} \mathrm{Ga}$ which lead to neutron-deficient $\mathrm{Rn}$ isotopes with the respective yields from the fragmentation reactions.

\section{MODEL}

Because the capture cross section is equal to the fusion cross section for the reactions ${ }^{A} \mathrm{Xe}+{ }^{48} \mathrm{Ca}$ treated in the present paper, the quantum diffusion approach [6, 7] for the capture is applied to study the complete fusion. With this approach many heavy-ion capture reactions at energies above and well below the Coulomb barrier have been successfully described [6 8]. Since the details of our theoretical treatment were already published in Refs. [6 8], the model will be only shortly described.

In the quantum diffusion approach [6, 7] the collisions of nuclei are described with a single relevant collective variable: the relative distance between the colliding nuclei. This approach takes into consideration the fluctuation and dissipation effects in collisions of heavy ions which model the coupling with various channels (for example, coupling of the relative motion with low-lying collective modes such as dynamical quadrupole and octupole modes of the target and projectile [9]). We have to mention that many quantum-mechanical and non-Markovian effects accompanying the passage through the Coulomb barrier are taken into consideration in our formalism [6-8]. The diffusion models, which include the quantum statistical effects, were also proposed in Refs. [10]. The nuclear deformation effects are taken into account through the dependence of the nucleus-nucleus potential on the deformations and mutual orientations of the colliding nuclei. To calculate the nucleus-nucleus interaction potential $V(R)$, we use the procedure presented in Ref. [7]. For the nuclear part of the nucleus-nucleus potential, the double-folding formalism with a Skyrme-type densitydependent effective nucleon-nucleon interaction is used [11]. The nucleon densities of the projectile and target nuclei are specified in the form of the Woods-Saxon parameterization, where the nuclear radius parameter is $r_{0}=1.15 \mathrm{fm}$ and the diffuseness parameter takes the values $a=0.55 \mathrm{fm}$ for all nuclei. The absolute values of the quadrupole deformation parameters $\beta_{2}$ of deformed nuclei were taken from Refs. [12] and [13] for the known and 
unknown nuclei, respectively. For the magic ${ }^{48} \mathrm{Ca}$ and semimagic ${ }^{136} \mathrm{Xe}$ nuclei in the ground state, we set $\beta_{2}=0$ and $\beta_{2}=0.05$, respectively.

The capture cross section is the sum of the partial capture cross sections [6, 7]

$$
\begin{aligned}
\sigma_{\text {cap }}\left(E_{\text {c.m. }}\right) & =\sum_{J} \sigma_{\text {cap }}\left(E_{\text {c.m. }}, J\right)= \\
& =\pi \lambda^{2} \sum_{J}(2 J+1) \int_{0}^{\pi / 2} d \theta_{1} \sin \theta_{1} \int_{0}^{\pi / 2} d \theta_{2} \sin \theta_{2} P_{\text {cap }}\left(E_{\text {c.m. }}, J, \theta_{1}, \theta_{2}\right),
\end{aligned}
$$

where $\lambda^{2}=\hbar^{2} /\left(2 \mu E_{\text {c.m. }}\right)$ is the reduced de Broglie wavelength, $\mu=m_{0} A_{1} A_{2} /\left(A_{1}+A_{2}\right)$ is the reduced mass ( $m_{0}$ is the nucleon mass), and the summation is over the possible values of the angular momentum $J$ at a given bombarding energy $E_{\text {c.m. }}$. Knowing the potential of the interacting nuclei for each orientation with the angles $\theta_{i}(i=1,2)$, one can obtain the partial capture probability $P_{\text {cap }}$ which is defined by the probability to penetrate the potential barrier in the relative distance coordinate $R$ at a given $J$. The value of $P_{\text {cap }}$ is obtained by integrating the propagator $G$ from the initial state $\left(R_{0}, P_{0}\right)$ at time $t=0$ to the final state $(R, P)$ at time $t(P$ is the momentum):

$$
\begin{aligned}
P_{\text {cap }} & =\lim _{t \rightarrow \infty} \int_{-\infty}^{r_{\text {in }}} d R \int_{-\infty}^{\infty} d P G\left(R, P, t \mid R_{0}, P_{0}, 0\right) \\
& =\lim _{t \rightarrow \infty} \frac{1}{2} \operatorname{erfc}\left[\frac{-r_{\text {in }}+\overline{R(t)}}{\sqrt{\Sigma_{R R}(t)}}\right] .
\end{aligned}
$$

Here, $r_{\text {in }}$ is an internal turning point. The second line in (2) is obtained by using the propagator $G=\pi^{-1}\left|\operatorname{det} \boldsymbol{\Sigma}^{-1}\right|^{1 / 2} \exp \left(-\mathbf{q}^{T} \boldsymbol{\Sigma}^{-1} \boldsymbol{q}\right)\left(\mathbf{q}^{T}=\left[q_{R}, q_{P}\right], q_{R}(t)=R-\overline{R(t)}, q_{P}(t)=\right.$ $P-\overline{P(t)}, \overline{R(t=0)}=R_{0}, \overline{P(t=0)}=P_{0}, \Sigma_{k k^{\prime}}(t)=2 \overline{q_{k}(t) q_{k^{\prime}}(t)}, \Sigma_{k k^{\prime}}(t=0)=0, k, k^{\prime}=$ $R, P)$ calculated for an inverted oscillator which approximates the nucleus-nucleus potential $V$ in the variable $R$ as follows. At given $E_{\text {c.m. }}$ and $J$, the classical action is calculated for the realistic nucleus-nucleus potential. Then the realistic nucleus-nucleus potential is replaced by an inverted oscillator which has the same barrier height and classical action. So, the frequency $\omega\left(E_{\text {c.m. }}, J\right)$ of this oscillator is set to obtain an equality of the classical actions in the approximated and realistic potentials. The action is calculated in the WKB approximation which is the accurate at the sub-barrier energies. Usually in the literature the parabolic approximation with $E_{\text {c.m. }}$-independent $\omega$ is employed which is not accurate at the deep sub-barrier energies. Our approximation is well justified for the reactions and energy range considered here [6, 7]. 
We assume that the sub-barrier capture mainly depends on the two-neutron transfer with positive $Q$-value. Our assumption is that, just before the projectile is captured by the target-nucleus (just before the crossing of the Coulomb barrier), the transfer occurs and leads to the population of the first excited collective state in the recipient nucleus [14]. So, the motion to the $N / Z$ equilibrium starts in the system before the capture because it is energetically favorable in the dinuclear system in the vicinity of the Coulomb barrier. For the reactions under consideration, the average change of mass asymmetry is connected to the two-neutron transfer. Since after the transfer the mass numbers, the isotopic composition and the deformation parameters of the interacting nuclei, and, correspondingly, the height $V_{b}=V\left(R_{b}\right)$ and shape of the Coulomb barrier are changed, one can expect an enhancement or suppression of the capture. If after the neutron transfer the deformations of the interacting nuclei increase (decrease), the capture probability increases (decreases). When the isotopic dependence of the nucleus-nucleus potential is weak and after the transfer the deformations of the interacting nuclei do not change, there is no effect of the neutron transfer on the capture. In comparison with Ref. [15], we assume that the negative transfer $Q$-values do not play a visible role in the capture process. Our scenario was verified in the description of many reactions [7].

The primary neutron-rich products of the complete fusion reactions ${ }^{A} \mathrm{Xe}+{ }^{48} \mathrm{Ca}$ of interest are excited and transformed into the secondary products with a smaller number of neutrons. Since neutron emission is the dominant deexcitation channel in the neutron-rich isotopes of interest, the production cross sections of the secondary nuclei are the same as those of the corresponding primary nuclei. This seems to be evident without special statistical treatment. The calculations are performed by employing the predicted values of the mass excesses and the neutron separation energies $S_{n}(Z, N)$ for unknown nuclei from the finite range liquid drop model [13].

\section{RESULTS OF THE CALCULATIONS}

\section{A. Complete fusion reactions ${ }^{A} \mathrm{Xe}+{ }^{48} \mathbf{C a}$}

To analyze the isotopic trend of the fusion cross section, it is useful to use the so called universal fusion function (UFF) representation [17]. The advantage of this representation 


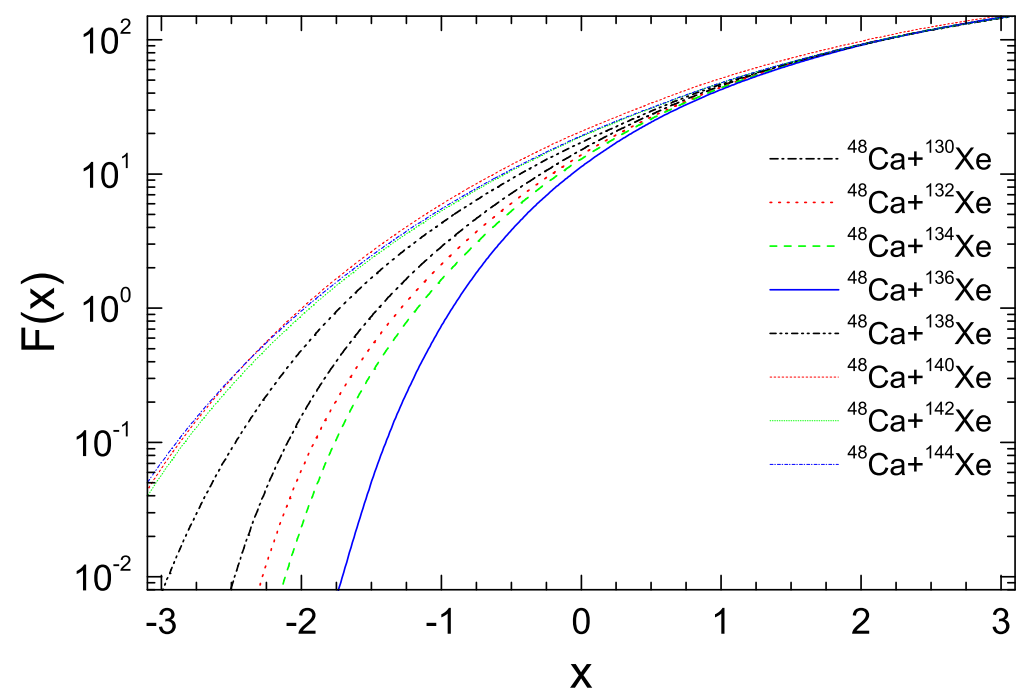

FIG. 1: (Color online) Calculated dependencies of $F(x)=\frac{2 E_{\text {c.m. }}}{\hbar \omega_{b} R_{b}^{2}} \sigma$ on $x=\frac{E_{\text {c.m. }}-V_{b}}{\hbar \omega_{b}}$ for the indicated reactions.

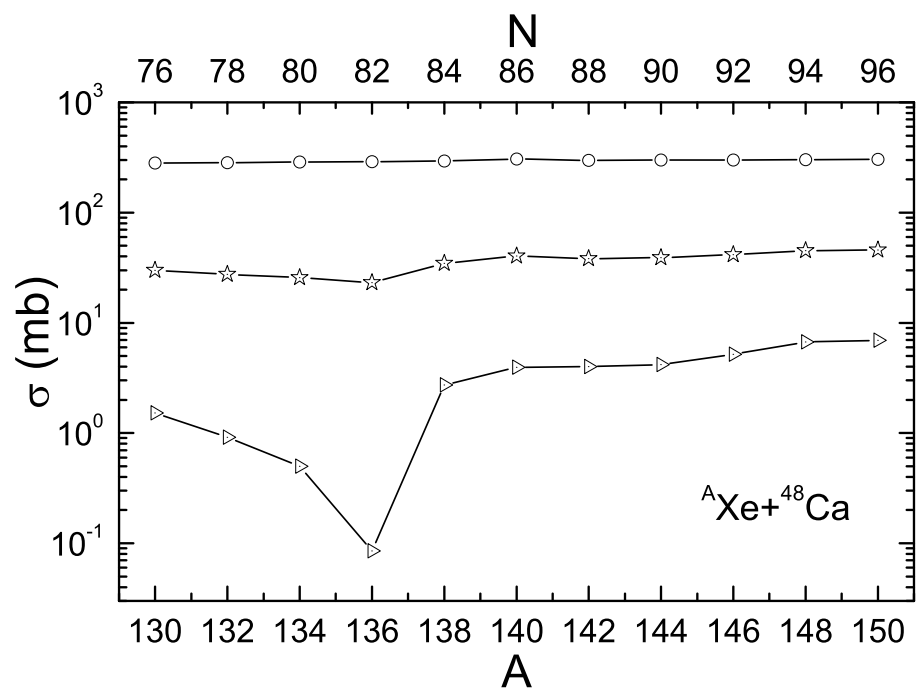

FIG. 2: Calculated dependence of fusion cross section $\sigma$ on $A$ for the reactions ${ }^{A} \mathrm{Xe}+{ }^{48} \mathrm{Ca}$ at fixed bombarding energies $E_{\text {c.m. }}=V_{b}-5 \mathrm{MeV}$ (triangles), $V_{b}$ (stars), $V_{b}+10 \mathrm{MeV}$ (circles).

appears clearly when one wants to compare fusion cross sections for systems with different Coulomb barrier heights and positions. In the reactions where the capture and fusion cross sections coincide, the elimination of the influence of the nucleus-nucleus potential on the fusion cross section with the UFF representation allows us to conclude about the role of deformation of the colliding nuclei and the nucleon transfer between interacting nuclei in the capture and fusion. In Ref. [17] the reduction procedure consists of the following 


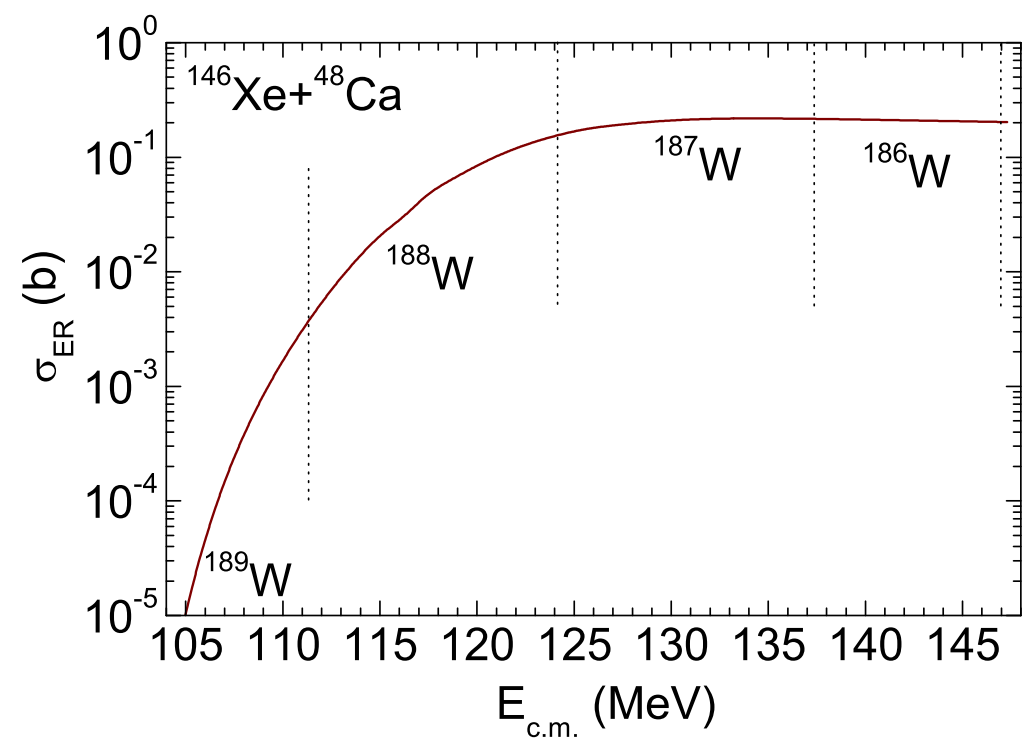

FIG. 3: The expected evaporation residue cross sections $\sigma_{E R}$ for the indicated neutron-rich isotopes ${ }^{186-189} \mathrm{~W}$ produced in the ${ }^{146} \mathrm{Xe}+{ }^{48} \mathrm{Ca}$ reaction. The vertical dashed lines show the range of energies for the production of given isotope.

transformations:

$$
E_{\text {c.m. }} \rightarrow x=\frac{E_{\text {c.m. }}-V_{b}}{\hbar \omega_{b}}, \quad \sigma \rightarrow F(x)=\frac{2 E_{\text {c.m. }}}{\hbar \omega_{b} R_{b}^{2}} \sigma
$$

The frequency $\omega_{b}=\sqrt{\left|V^{\prime \prime}\left(R_{b}\right)\right| / \mu}$ is related with the second derivative $V^{\prime \prime}\left(R_{b}\right)$ of the nucleus-nucleus potential $V(R)$ at the barrier radius $R_{b}$ and the reduced mass parameter $\mu$. With these replacements one can compare the cross sections for different reactions.

In Fig. 1, one can see the comparison of the calculated functions $F(x)$ for the reactions $130,132,134,136,138,140,142,144 \mathrm{Xe}+{ }^{48} \mathrm{Ca}$ with stable and radioactive beams. As expected, at sub-barrier energies the enhancement of the complete fusion (capture) cross section is larger in the case of reactions with strongly quadrupole deformed projectile-nuclei and after neutron transfer. The quadrupole deformation parameter $\beta_{2}$ of the projectile nucleus increases with changing mass number from $A=136$ to $A=130$ or to $A=144$. For the reaction ${ }^{136} \mathrm{Xe}+{ }^{48} \mathrm{Ca}$ with spherical target and projectile and without neutron transfer the cross section is the smallest one at $x<0$. The sub-barrier cross sections for the reactions $138,140,142,144,146,148,150 \mathrm{Xe}+{ }^{48} \mathrm{Ca}$ with neutron transfer (positive $Q$-values) are larger than those for the reactions ${ }^{130,132,134,136} \mathrm{Xe}+{ }^{48} \mathrm{Ca}$, where the neutron transfer is suppressed (negative $Q$-values). Since after two-neutron transfer the mass numbers and the deformation param- 


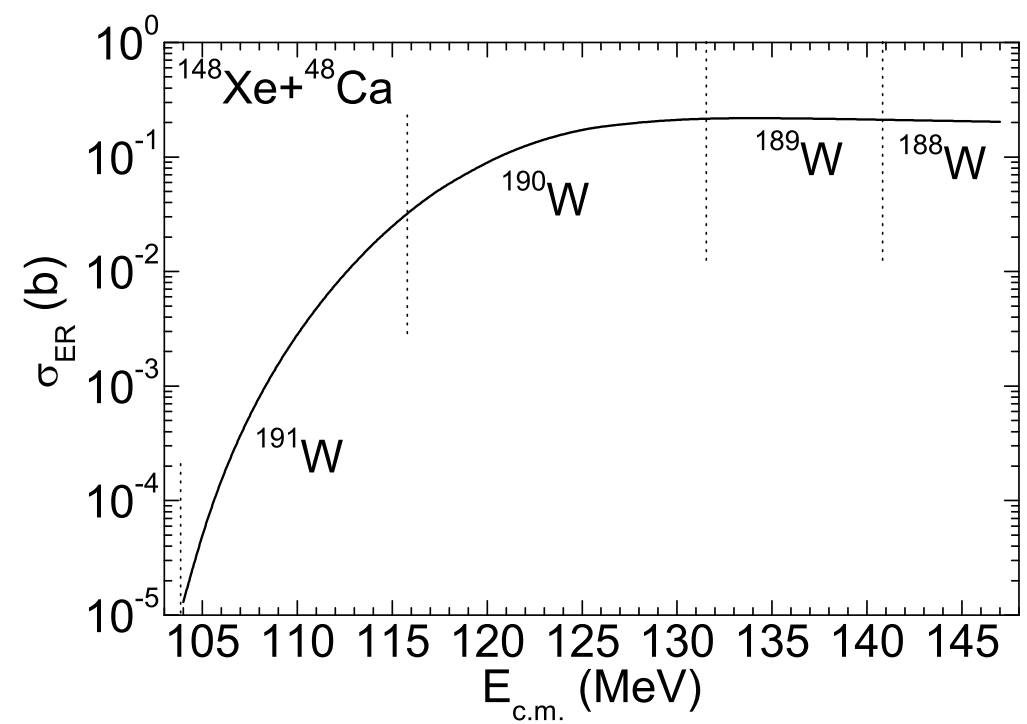

FIG. 4: The expected evaporation residue cross sections $\sigma_{E R}$ for the indicated neutron-rich isotopes ${ }^{188-191} \mathrm{~W}$ produced in the ${ }^{148} \mathrm{Xe}+{ }^{48} \mathrm{Ca}$ reaction. The vertical dashed lines show the range of energies for the production of given isotope.

eters of the interacting nuclei are changed and the height of the Coulomb barrier decreases, one can expect an enhancement of the capture. For example, after the neutron transfer in the reaction ${ }^{144} \mathrm{Xe}\left(\beta_{2}=0.18\right)+{ }^{48} \mathrm{Ca}\left(\beta_{2}=0\right) \rightarrow{ }^{142} \mathrm{Xe}\left(\beta_{2}=0.15\right)+{ }^{50} \mathrm{Ca}\left(\beta_{2}=0.25\right)$, the deformation of the target-nucleus increases and the mass asymmetry of the system decreases, and, thus, the value of the Coulomb barrier decreases and the capture cross section becomes larger (Fig. 1). We observe the same behavior in the reactions with the projectiles $138,140,142,146,148,150$ Xe.

The complete fusion (capture) cross sections for the reactions $130,132,134,136,138,140,142,144,146,148,150 \mathrm{Xe}+{ }^{48} \mathrm{Ca}$ at different bombarding energies are presented in Fig. 2. The behaviour of the curves in Fig. 2 is determined by the quadrupole deformation and neutron transfer effects. The isotopic dependency is rather weak at energies above the corresponding Coulomb barriers. At sub-barrier energies the fusion cross section decreases by about one order of magnitude with increasing mass number $A$ of the projectile from $A=130$ up to $A=136(N=82)$. For $A>136$ a steep increase can be observed for beam energies of $5 \mathrm{MeV}$ below the corresponding Coulomb barriers. At energies near the Coulomb barrier the cross section changes in a similar way but the curve shows a much flatter slope. 


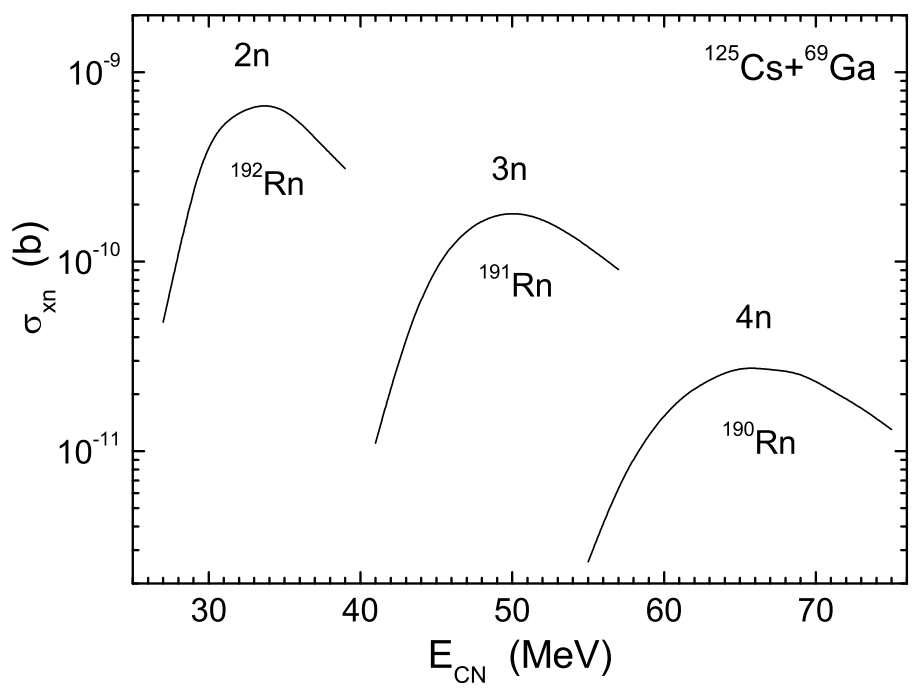

FIG. 5: The expected evaporation residue cross sections $\sigma_{x n}$ for the indicated neutron-deficient isotopes of $\mathrm{Rn}$ produced in the $x n$-channels $(x=2-4)$ of the ${ }^{125} \mathrm{Cs}+{ }^{69} \mathrm{Ga}$ reaction.

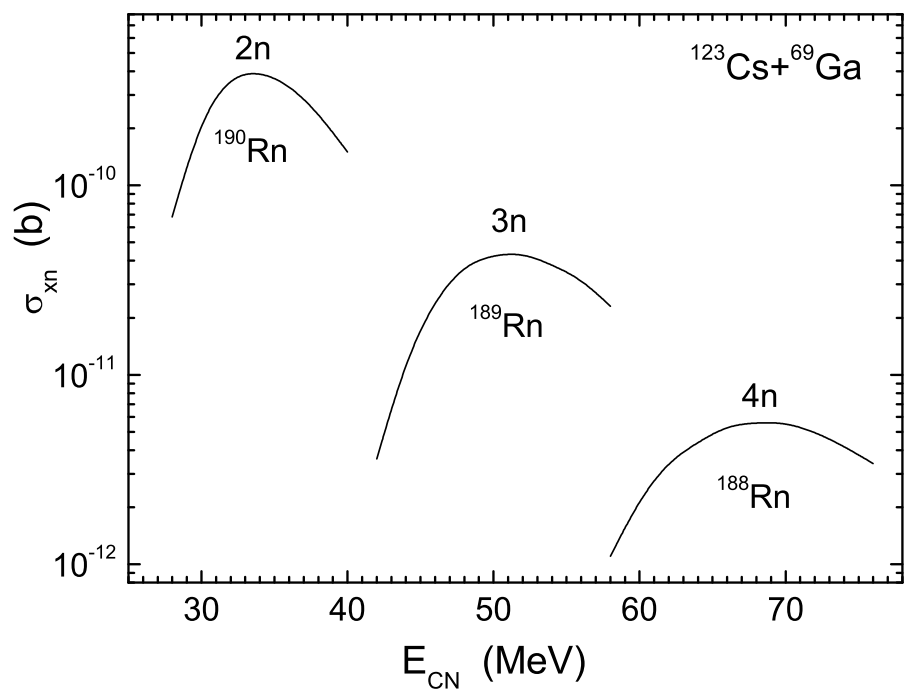

FIG. 6: The same as in Fig. 5, but for the ${ }^{123} \mathrm{Cs}+{ }^{69} \mathrm{Ga}$ reaction.

In Figs. 3 and 4 we present the possibilities for future experiments to produce the neutronrich isotopes ${ }^{186-191} \mathrm{~W}$ in complete fusion reactions of ${ }^{146,148} \mathrm{Xe}+{ }^{48} \mathrm{Ca}$ with radioactive beams. The production cross sections of the neutron-rich ${ }^{190,191} \mathrm{~W}$ isotopes, for example, are between the $10 \mu \mathrm{b}$ and $100 \mathrm{mb}$ levels meaning that they can be observed with rather low beam intensities and with the present experimental techniques. The calculated cross sections are more than two orders of magnitude larger than in fragmentation reactions [18]. Note also, that when the neutron number approaches the drip-line the production cross section in 
complete fusion decreases not so fast as in fragmentation reactions.

\section{B. Comparison between complete fusion and fragmentation reactions}

The availability of heavy radioactive beams at Coulomb barrier energies at future facilities like FAIR, HIE-ISOLDE or SPIRAL-II will enable the experimental utilization of the above discussed effects for fusion reactions. Another competing method to produce heavy exotic isotopes is projectile fragmentation at relativistic energies which is for example used at the Fragment Separator (FRS) at GSI. In the following, we give some comparative considerations on both methods since, depending on the region of the nuclear chart, fragmentation can lead to high yields of exotic nuclei. As an example, we consider the isotope ${ }^{189} \mathrm{~W}$. Cross-sections of up to about $2 \mathrm{mb}$ are predicted for its production in the complete fusion reactions of ${ }^{146} \mathrm{Xe}+{ }^{48} \mathrm{Ca}$ at $\mathrm{E}_{c m}=110 \mathrm{MeV}$. Cross-sections on the same order are also measured in the fragmentation reactions leading to yields of $10^{4}$ ions/s. At the future Super-FRS facility even yields of $2 \times 10^{6}$ ions/s are predicted. In order to obtain at least the same yields of $10^{4}$ ions/s in fusion reactions, ${ }^{146} \mathrm{Xe}$ beams with intensities of at least $10^{13}$ ions/s are required. The largest intensities for neutron-rich Xe beams are expected at SPIRAL-II where $10^{5}$ of ${ }^{146}$ Xe projectiles per second are predicted which is, however, still eight orders of magnitude less than needed for an efficient application of fusion reactions to reach ${ }^{189} \mathrm{~W}$.

As an other example, we discuss in the following the synthesis of neutron deficient $\mathrm{Rn}$ $(Z=86)$ isotopes in the complete fusion reactions. Figures 5 and 6 show the calculated excitation functions for fusion reactions of ${ }^{123,125} \mathrm{Cs}$ beams with ${ }^{69} \mathrm{Ga}$ target. The survival probabilities of the excited compound nuclei in the neutron evaporation channels $x n(x=$ $2-4)$ are calculated by employing the modified statistical code GROGIF [19] with the same parameters as in Ref. [20]. The capture cross sections and fusion probabilities are calculated with the quantum diffusion approach [6, 7] and the dinuclear system fusion model [20], respectively. Radioactive Cs beams are already now available with high intensities for a broad variety of isotopes and are therefore favourable projectiles. At REX-ISOLDE for example, the isotopes ${ }^{122-129} \mathrm{Cs}$ are provided with intensities around $10^{10} \mathrm{ions} / \mathrm{s}$ and for the future HIE-ISOLDE facility ten times higher intensities are expected at beam energies of $\geq$ $5.5 \mathrm{MeV} /$ nucleon. A comparison of the predicted yields for neutron deficient Rn isotopes at the SuperFRS facility with the expected yields from fusion evaporation reactions with ${ }^{123} \mathrm{Cs}$ 
beams at intensities of $10^{10}$ ions/s leads to the conclusion that the complete fusion is not superior to fragmentation for ${ }^{A} \mathrm{Rn}$ isotopes with $188 \leq A \leq 190$. For these mass numbers at least 2-7 times lower yields can be obtained in the fusion reactions with the presently available beam intensities.

\section{SUMMARY}

Because of deformation and neutron transfer effects, a strong dependence of the subbarrier complete fusion (capture) cross section on the isospin was found for the reactions ${ }^{130,132,134,136,138,140,142,144,146,148,150} \mathrm{Xe}+{ }^{48} \mathrm{Ca}$. At fixed bombarding energy, the cross section increases with changing mass number of the projectile-nucleus from $A=136$ to $A=130$ or to $A=150$. The ${ }^{136} \mathrm{Xe}+{ }^{48} \mathrm{Ca}$ reaction with magic and semimagic nuclei has the smallest cross section. The complete fusion (capture) cross sections for the reactions ${ }^{130,132,134,136} \mathrm{Xe}+{ }^{48} \mathrm{Ca}$ without neutron transfer are smaller than those for the reactions ${ }_{138,140,142,144,146,148,150} \mathrm{Xe}+{ }^{48} \mathrm{Ca}$ with neutron transfer. We demonstrated the possibilities for producing neutron-rich isotopes of ${ }^{186-191} \mathrm{~W}$ with relatively large cross sections for future experiments in the complete fusion reactions ${ }^{146,148} \mathrm{Xe}+{ }^{48} \mathrm{Ca}$ with radioactive beams. However, we found that for the production of neutron-rich $\mathrm{W}$ the fragmentation reactions are more preferable than the complete fusion reactions. Even if we consider here the formation of neutron-rich $\mathrm{W}$ isotopes as an example, our findings have general validity and are not restricted to specific isotopes. Exotic nuclei with large deformations which could be used as projectiles can equally be found in wide regions on the neutron-rich as well as on the neutron-deficient side of the nuclear chart.

We concluded also that the complete fusion ${ }^{123} \mathrm{Cs}+{ }^{69} \mathrm{Ga}$ reaction with radioactive beam

${ }^{123} \mathrm{Cs}$ is not superior to fragmentation for the production of neutron-deficient isotopes of ${ }^{188-190} \mathrm{Rn}$. The fragmentation reactions result in slightly larger yields of these isotopes. Note that the choice of the method of production of the isotopes near the drip lines would be also affected by the purposes of the experiments and the available facilities.

This work was supported in part by DFG and RFBR. The IN2P3(France)-JINR(Dubna) 
and Polish - JINR(Dubna) Cooperation Programmes are gratefully acknowledged.

[1] W. Loveland, Phys. Rev. C 76, 014612 (2007); ibid 75, 069801 (2007).

[2] P.E. Haustein, E.M. Franz, S. Katcoff, N.A. Morcos, H.A. Smith, Jr., and T.E. Ward, Phys. Rev. C 14, 645 (1976).

[3] J. Benlliure, K.-H. Schmidt, D. Cortina-Gil, T. Enqvist, F. Farget, A. Heinz, A.R. Jurghans, J. Pereira, and J. Taieb, Nucl. Phys. A660, 87 (1999).

[4] Zs. Podolyak et al., Phys. Lett. B 491, 255 (2000).

[5] J. Kurcewicz et al., Phys. Lett. B 717, 371 (2012).

[6] V.V. Sargsyan, G.G. Adamian, N.V. Antonenko, and W. Scheid, Eur. Phys. J. A 45, 125 (2010); V.V. Sargsyan, G.G. Adamian, N.V. Antonenko, W. Scheid, and H.Q. Zhang, Eur. Phys. J. A 47, 38 (2011); J. of Phys.: Conf. Ser. 282, 012001 (2011); EPJ Web Conf. 17, 04003 (2011); V.V. Sargsyan, G.G. Adamian, N.V. Antonenko, W. Scheid, C.J. Lin, and H.Q. Zhang, Phys. Rev. C 85, 017603 (2012); Phys. Rev. C 85, 037602 (2012).

[7] V.V. Sargsyan, G.G. Adamian, N.V. Antonenko, W. Scheid, and H.Q. Zhang, Phys. Rev. C 84, 064614 (2011); Phys. Rev. C 85, 024616 (2012).

[8] R.A. Kuzyakin, V.V. Sargsyan, G.G. Adamian, N.V. Antonenko, E.E. Saperstein, and S.V. Tolokonnikov, Phys. Rev. C 85, 034612 (2012).

[9] S. Ayik, B. Yilmaz, and D. Lacroix, Phys. Rev. C 81, 034605 (2010).

[10] H. Hofmann, Phys. Rep. 284, 137 (1997). S. Ayik, B. Yilmaz, A. Gokalp, O. Yilmaz, and N. Takigawa, Phys. Rev. C 71, 054611 (2005); V.V. Sargsyan , Z. Kanokov, G.G. Adamian, and N.V. Antonenko, Part. Nucl. 41, 175 (2010); G. Hupin and D. Lacroix, Phys. Rev. C 81, 014609 (2010).

[11] G.G. Adamian et al., Int. J. Mod. Phys. E 5, 191 (1996).

[12] S. Raman, C.W. Nestor, Jr, and P. Tikkanen, At. Data Nucl. Data Tables 78, 1 (2001).

[13] P. Möller et al., At. Data Nucl. Data Tables 59, 185 (1995).

[14] S. Szilner et al., Phys. Rev. C 76, 024604 (2007); S. Szilner et al., Phys. Rev. C 84, 014325 (2011); L. Corradi et al., Phys. Rev. C 84, 034603 (2011).

[15] C.H. Dasso, S. Landowne, and A. Winther, Nucl. Phys. A405, 381 (1983).

[16] G.G. Adamian, A.K. Nasirov, N.V. Antonenko, and R.V. Jolos, Phys. Part. Nucl. 25, 583 
(1994); K. Washiyama, D. Lacroix, and S. Ayik, Phys. Rev. C 79, 024609 (2009); S. Ayik, K. Washiyama, and D. Lacroix, Phys. Rev. C 79, 054606 (2009).

[17] L.F. Canto, P.R.S. Gomes, J. Lubian, L.C. Chamon, and E. Crema, J. Phys. G 36, 015109 (2009); Nucl. Phys. A821, 51 (2009).

[18] J. Benlliure et al., Nucl. Phys. A 660, 87 (1999).

[19] J. Gilat, Phys. Rev. C 1, 1432 (1970); O.V. Grusha et al., Nucl. Phys. A429, 313 (1984); O.V. Grusha, S.P. Ivanova, and Yu.N. Shubin, VANT, Nuclear Constants 1, 36 (1987); A.S. Zubov, G.G. Adamian, N.V. Antonenko, S.P. Ivanova, and W. Scheid, Phys. Rev. C 68, 014616 (2003).

[20] G.G. Adamian, N.V. Antonenko, W. Scheid, and A.S. Zubov, Phys. Rev. C 78, 044605 (2008). 